desde la academia

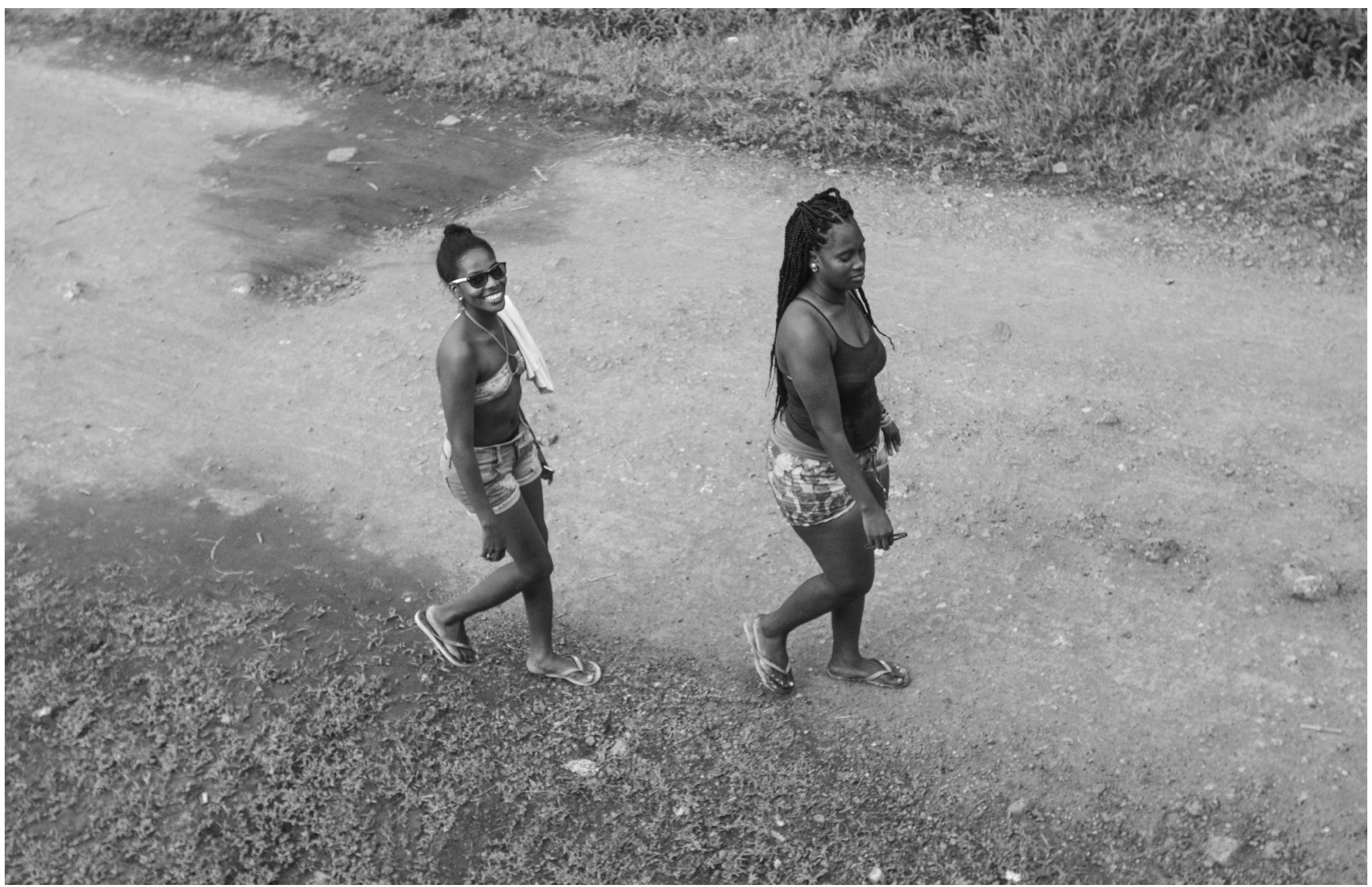

Foto: Cecilia Vidal

\title{
El diagnóstico de injusticias para el enmarcamiento social. Una aproximación a los procesos organizativos de la Asociación de Mujeres Afrocolombianas de la ciudad de Cali
}

The diagnosis of injustices for the social framing. An approach to the organizative processes of the Afro-Colombian Women's Association of the city of Cali

DOI: https://doi.org/10.22235/d.v0i30.1794

Sofía Arias Alonso y Carlos Tobar Tovar 
El propósito de este artículo es analizar los procesos organizativos de grupos históricamente subordinados que se derivan del diagnóstico de las injusticias para la cimentación de una identidad colectiva y que, por ello, contribuyen al enmarcamiento social. Se toma como caso de estudio una agremiación de mujeres afrocolombianas de la ciudad de Cali que apuestan a la educación y a la reivindicación de las estéticas femeninas afro para crear discursos que les permitan subvertir estas injusticias. El trabajo de campo y la realización de entrevistas semiestructuradas permitieron identificar los procesos organizativos que dan lugar al diagnóstico y analizar cómo estos dan lugar a prácticas de enmarcamiento social que permiten describir experiencias de liderazgo o agenciamiento. El estudio de caso revela el papel determinante que tiene la percepción de las injusticias para el proceso organizativo y el modo en que la experiencia del enmarcamiento hace posible la aparición de prácticas de agenciamiento comunitario.

Palabras clave: injusticias; enmarcamiento social; identidad colectiva; afrodescendientes; organización femenina.
The purpose of this article is to analyze the organizative processes of historically subordinated groups that derive from the diagnosis of injustices for the foundation of a collective identity and therefore contribute to social framing. As a case of study, we analyze an association of Afro-Colombian women from the city of Cali who believe both in education and in vindicating Afro-feminine aesthetics to create discourses that allow them to subvert these injustices. Fieldwork and semi-structured interviews made possible the identification of the organizative processes that lead to the diagnosis and the analysis of how these result in social framing practices that allow us to describe leadership or agency experiences. The case study reveals the determinant role that the perception of injustices plays for the organizational process and the way in which the framing experience makes possible the appearance of communitarian agency practices.

Keywords: injustices; social framing; collective identity; afrodescendants; female organization.

para el estudio de estos grupos - que han aprendido que el proceso organizativo les permite una mejor posición estratégica para la lucha social en contextos violentos- la constituye el enmarcamiento social. Siguiendo a Villafuerte (2008), los procesos de enmarcamiento refieren a "esfuerzos estratégicos conscientes realizados por grupos de personas para construir interpretaciones compartidas del mundo y de sí mismos que legitiman y motivan la acción colectiva” (p. 241).

Se estima que las acciones derivadas de estos procesos llevan tras de sí la impronta de las expectativas del cambio social que se forjan cuando se ha avanzado en una comprensión de las injusticias padecidas grupalmente y que tienen un efecto en la disminución de oportunidades sociales relativas al reconocimiento cultural, la redistribución económica y la representación política (Fraser, 1997). El enmarcamiento social permite vislumbrar cómo "los movimientos sociales tratan de construir un discurso coherente que permita definir los problemas por los que luchan, las causas que los originaron y las soluciones y estrategias adecuadas para enfrentarlos" (Chihu y López, 2004, p.449).
Sofía Arias Alonso Pontificia Universidad Javeriana Cali, Colombia. https://orcid.org/00000001-9818-647X sofiarias0805@gmail.com

Carlos Tobar Tovar Pontificia Universidad Javeriana Cali, Colombia. https://orcid.org/00000001-5144-5462 catobar@javerianacali.edu.co

Recepción: 21/03/2019 Aceptación: 30/04/2019 
Es preciso enfatizar el carácter estratégico que deviene del modo de interpretar el afrontamiento de las luchas sociales. De ahí que los procesos de comunicación orientados al cambio social deban estar situados dentro de las experiencias reconocidas en las que el grupo fundamenta posibilidades de éxito (Berrío Puerta, 2006). De este modo, el enmarcamiento social tiene lugar en formas de objetivación de las luchas sociales, en el entendido de que tales procesos son generadores de significados que definen y dan sentido a las identidades que van emergiendo. Dicho de otra manera, la participación en los conflictos sociales produce identidades colectivizadas (Clastres, 1977).

En este orden de ideas, el análisis de los procesos de organización, fundamentados en el enmarcamiento social, apelan a las experiencias comunicativas que dan sentido a una gramática moral capaz de nombrar tanto el diagnóstico de las injusticias como las vías normativas que componen el cambio social (Honneth, 1997). Se trata de una forma de otorgar sentido al lenguaje que se emplea en las disputas por el poder, por la capacidad de nombrar desde el punto de vista del subordinado una manera valida de concebir el afrontamiento de la conflictividad social.

Desde esta perspectiva, la presente investigación se enfoca en el análisis de los procesos organizativos de la Asociación de Mujeres Afrocolombianas (Amafrocol) a través de uno de sus emprendimientos más visibles: la peluquería boutique-afro Makeda. Esta organización forma parte del entramado de luchas sociales promovidas por los movimientos afrodescendientes y las colectividades feministas que habitan la ciudad de Cali. El estudio se orienta desde la pregunta acerca del alcance que obtiene el enmarcamiento social en el ámbito de la experiencia política que proponen estas mujeres.

Es necesario detenerse en los dos aspectos que suelen orientar la mirada sobre las prácticas organizativas de estos grupos, y que fueron mencionados al inicio de este artículo. Aplicado al caso de estudio, se entiende que, por una parte, la inclusión de las mujeres afrocolombianas al ámbito del reconocimiento estatal responde al modo en que han aprendido a ser visibles y representarse políticamente en la discusión pública de los criterios de justicia social. Es decir, han generado aprendizajes que les permiten considerar que organizarse en colectividades es el mejor modo de complementar la lucha social aprovechando las líneas jurisprudenciales derivadas de los Fallos de las Altas Cortes y la Constitución Política de 1991 (Comins Mingol, 2002). Por otra parte, las mujeres que integran el objeto de estudio provienen de regiones azotadas por el conflicto armado colombiano y su agremiación responde a una manera de integrarse a la vida urbana a través de la aproximación a lenguajes y estéticas relacionadas con prácticas y reflexiones propias de los movimientos sociales afrocolombianos y la perspectiva de género aportada por el feminismo.

Como se verá más adelante, muchas de las participantes entrevistadas manifestaron ser víctimas del conflicto armado dado que sus familias provienen de la región pacífica, un contexto caracterizado por la incursión reiterada de grupos alzados en armas. Tal incursión tendría efectos en las posibilidades de articulación de los grupos sociales a la vida política, ya que el ordenamiento de la convivencia por la vía violenta causó una desposesión de derechos y, especialmente, un desplazamiento forzado (Moncayo, 2015).

Por estas razones, se entiende que el esfuerzo que realiza este grupo por diagnosticar injusticias está estrechamente asociado con las prácticas de comunicación en las que sus participantes proponen su visión sobre cómo el racismo y el machismo limitan su existencia en las esferas de la cultura, la economía y la política. En esta experiencia, diagnosticar supone tomar conciencia respecto de cómo la pobreza, la misoginia y la estigmatización constituyen limitaciones en la vida social. Según el enfoque propuesto en esta investigación, este proceso de diagnóstico es el que da lugar al enmarcamiento social. 
La Asociación de Mujeres Afrocolombianas

y Makeda como caso de estudio

Amafrocol se constituyó en 1997 como un espacio para la discusión sobre las injusticias que padecen las mujeres afrocolombianas y, en particular, para la reivindicación de las expectativas culturales de reconocimiento en el marco de experiencias de discriminación que han alterado la imagen de las mujeres.

Esta organización se ubica en la Comuna 4 de la ciudad de Cali y si bien su proyección social está orientada a diferentes contextos de la ciudad, poseen una trayectoria importante de procesos de intervención en la Comuna 21 del Distrito de Aguablanca. Amafrocol desarrolla ejercicios de sensibilización sobre la vulnerabilidad que padecen las mujeres afrodescendientes, entre los que se destacan las retóricas alusivas al empoderamiento político y la reivindicación de los procesos sociales afrodescendientes en los sectores marginados de la ciudad. Se trata de un grupo que cuenta con una trayectoria relevante de trabajo social y cuyas participantes entienden el escenario de la formación universitaria como un camino para perfeccionar las estrategias de lucha social.

Uno de los resultados del esfuerzo organizativo de las integrantes de Amafrocol se materializó en Makeda, emprendimiento reconocido como la primera peluquería boutique-afro de Cali y del país. A través de Makeda se promueve el empoderamiento y la autonomía de las mujeres por medio de la cualificación laboral y la generación de emprendimientos. Además, la peluquería participa en eventos culturales relacionados con las comunidades afrocolombianas, como el Festival de Música del Pacífico Petronio Álvarez. Esta es una experiencia cultural de enorme importancia para las comunidades afrodescendientes de departamentos del Valle del Cauca, Chocó y Nariño. En este evento, las mujeres realizan los peinados tradicionales de la región y relatan su relevancia ante diversos públicos ajenos a la cuestión afro. Así, Makeda se ha convertido en un medio para la difusión de mensajes relativos al valor cultural de la experiencia de las mujeres afro en la ciudad de Cali.

Puede observarse cómo en el trabajo de esta organización toma especial importancia la dimensión estética como una postura política y un símbolo de resistencia. Esto se materializa en la discusión sobre los modos de peinar el cabello y cómo representar la expresividad afrocolombiana al recuperar las estéticas femeninas típicas de la región del Pacífico. El cuerpo de la mujer es, entonces, ponderado en aras de posicionar un discurso político a favor del reconocimiento cultural de la experiencia femenina en las zonas de influencia de Amafrocol. Se centra la atención sobre cómo las estéticas autóctonas pueden ayudar a representar el contenido de las demandas de este grupo ante otros grupos y ante el Estado.

\section{Aspectos metodológicos}

Este trabajo se inscribe en el semillero de investigación titulado "Enmarcamiento Social de Movimientos Culturales: una Aproximación a los Grupos Negativamente Diferenciados" de la Pontificia Universidad Javeriana de Cali. Este escenario de formación se fundó con el ánimo de generar una discusión sobre la construcción de la paz en el departamento del Valle del Cauca a partir de experiencias etnográficas con grupos en condición de vulnerabilidad social. ${ }^{1}$

En este marco, el presente artículo se sustenta en un estudio de caso realizado entre junio de 2018 y febrero de 2019 a través del encuentro con las mujeres de Amafrocol agremiadas en Makeda. Se trata de un grupo compuesto por 25 integrantes activas (de un total de 65) que tiene entre sus líderes a Emilia Valencia, quien realizó un proceso de formación política en la Universidad del Valle. ${ }^{2}$

Para el desarrollo de esta investigación se realizaron observaciones y visitas semanales a la organización, y se participó activamente en las actividades de proyección social del grupo durante el tiempo que duró
1:: Los semilleros de investigación refieren a agrupaciones de enseñanza y aprendizaje compuestas por estudiantes, profesores y líderes sociales para desarrollar competencias investigativas a través del estudio de procesos socialmente relevantes para el desarrollo del departamento del Valle del Cauca.

2:: Entidad pública de educación superior que nuclea muchas de las reflexiones sobre los movimientos sociales y su impacto en la región. 
el estudio. De esta forma se alcanzó el grado de acceso y confianza suficientes para proponer dinámicas de encuentro que favorecieron el registro de datos y, en términos generales, la elaboración del trabajo de campo. Este trabajo de campo se complementó con la aplicación de entrevistas semiestructuradas a las participantes.

Como se mencionó más arriba, el enfoque del trabajo toma como punto de partida el diagnóstico de las injusticias y la consolidación de un horizonte de cambio social como elementos que favorecen prácticas de agenciamiento o liderazgo al interior de grupos históricamente estigmatizados. Para cumplir con este propósito, se analizó el caso de estudio teniendo en cuenta tres factores:

- La identificación de los procesos organizativos que dieron lugar al diagnóstico.

- El análisis de cómo tales procesos dan lugar a prácticas de enmarcamiento social.

- La descripción de las experiencias de liderazgo que hacen posible la subsistencia de la organización.

El procedimiento metodológico se desprende de estas tres proposiciones; la investigación se realizó acompañando al grupo para dar cuenta del modo en que llevan a cabo las diferentes prácticas relativas al diagnóstico de las injusticias, el enmarcamiento social y el agenciamiento. El análisis propuesto aquí responde a esta estructura, que coincide con la manera en que se han realizado estudios previos cercanos a estos temas (Tobar Tovar, 2015; Rodríguez, 2015).

Esta manera de reflexionar sobre la organización social sugiere que las luchas sociales son dinámicas y generadoras de identidad (Torrico Villanueva, 2004). En definitiva, se entiende que la participación en el entramado organizativo que se requiere para afrontar la lucha social posibilita la concreción de una identidad colectiva capaz de producir intercambios y determinar los lenguajes con los que se nombra la convivencia en la diferencia (Chaparro Escudero, 2015).

A continuación, se presentan los ejes del análisis propuesto. En primer término, se alude al papel que tiene la percepción o la conciencia sobre las injusticias en la delimitación de las estrategias que dan lugar al proceso organizativo de Amafrocol. En segundo término, se expondrá el modo en que la experiencia del enmarcamiento social da lugar a prácticas de agenciamiento o liderazgo comunitario que dimensionan la proyección social de este grupo.

\section{El diagnóstico de las injusticias}

y el proceso organizativo

Los procesos de enmarcamiento social son formas elaboradas de estructuración de un lenguaje sobre lo que se considera injusto y las vías para generar su afrontamiento. Siguiendo a Fraser (1996), las formas en que las injusticias afectan a los individuos están relacionadas con el mal reconocimiento de sus identidades, la inequitativa distribución económica y las limitadas posibilidades de representación política. La autora señala el sustrato político que estructura los problemas de distribución y reconocimiento, y los articula con la exclusión de las instancias en las que se deciden asuntos relevantes para la organización social. De ahí que la injusticia sea un concepto tridimensional, ya que supone formas complejas de limitaciones a la vida social en los campos de la cultura, la economía y la política (Bourdieu, 2010). El diagnóstico que realizan los grupos sobre sus vicisitudes corresponde al enfoque de estos tres campos.

\section{La dimensión cultural}

Dentro de esta dimensión entran las injusticias que refieren al efecto que tiene la discriminación en la valoración social de los grupos y que dan lugar a experiencias de reivindicación. En este caso se destacan los relatos alusivos al rechazo y la ausencia de reco- 
nocimiento respecto de los dramas sociales de quienes padecen vulnerabilidades. Son posturas que aluden a formas de inferioridad materializadas en diversas expresiones de la vida cultural de los grupos, el rechazo a las tradiciones y a la valoración de las costumbres como regresivas o descontextualizadas del ámbito del desarrollo nacional (Elias, 1998).

En este sentido, una experiencia de injusticia es aquella en la que los grupos son sometidos a patrones de interpretación correspondientes a culturas ajenas que son hostiles a las formas de vida de las comunidades menos cohesionadas o con menor grado de desarrollo institucional. Esta forma de dominación cultural se expresa en términos de falta de respeto; de ahí que la difamación sea una práctica de menoscabo que funciona a través del ejercicio sistemático de la mentira y la promoción de estereotipos que afectan la interacción cotidiana (Honneth, 2011). En palabras de una de las participantes:

Hay aspectos que nos reconocen, pero esos mismos nos estigmatizan y discriminan. No somos solo baile y gastronomía. Algo que me encanta destacar de la cultura negra es que somos personas muy capaces, fuertes. Tenemos resiliencia, adaptabilidad, poder superar muchas cosas que nos quieren dejar en el camino. Somos muy inteligentes, buenos compañeros. Si vemos que alguien está cayendo, es tratar de cogerlo (Participante de Amafrocol, comunicación personal, 4 de agosto de 2018).

En el relato se puede apreciar cómo uno de los aspectos que compone el mal reconocimiento refiere a la ponderación de costumbres "aceptables" - como la jovialidad y la gastronomía de un grupo- en detrimento de las posibilidades de construcción de procesos organizativos, los cuales complejizan la manera de considerar los dramas de las negritudes en el Valle del Cauca. La valoración parcial de la vida cultural constituye una forma de legitimar prácticas de dominación que termi- nan siendo armonizables con los horizontes dispuestos por las hegemonías para legitimar la aceptabilidad de los conflictos interculturales (Gledhill, 2000).

Entonces, la valoración de los atributos de las prácticas culturales por encima de las posibilidades organizativas corresponde al modo en que los grupos hegemónicos clasifican los aspectos aceptables de la diversidad cultural. Se valoran los atributos menos controversiales de la vida cultural, pero no aquellos que tienen el potencial de cuestionar el entramado de desigualdades que legitiman una convivencia injusta hacia los grupos étnicamente diferenciados. Es importante llamar la atención al modo en que las mujeres participantes del estudio refieren a este problema, puesto que parte de la cosificación del cuerpo femenino afrodescendiente pasa por el modo en que se legitima su presencia: la necesidad de adaptar el cuerpo a las expectativas de un sistema racista, machista y misógino también corresponde a los criterios de inclusión y exclusión resultantes de la percepción de la diversidad cultural.

Se resalta la alusión al uso del cabello y la necesidad de limitar o adaptar su expresión a las expectativas de los grupos circunscritos en estéticas hegemónicas. De ahí que la existencia de una estética de la subordinación -que se corresponde con una manera socialmente aceptada de percibir a la mujer afro sea un asunto que se preste para el análisis desde la perspectiva del diagnóstico de las injusticias.

\section{La dimensión económica}

En esta dimensión aparecen las injusticias circunscritas al ámbito de la inadecuada redistribución del capital. Siguiendo a Fraser (2008), es importante resaltar esta dimensión porque es en ella donde la injusticia se hace más evidenciable. La falta de ingresos, la situación de subempleo y la explotación constituyen problemáticas que limitan la capacidad que tienen los individuos para desarrollar su proyecto de vida. 


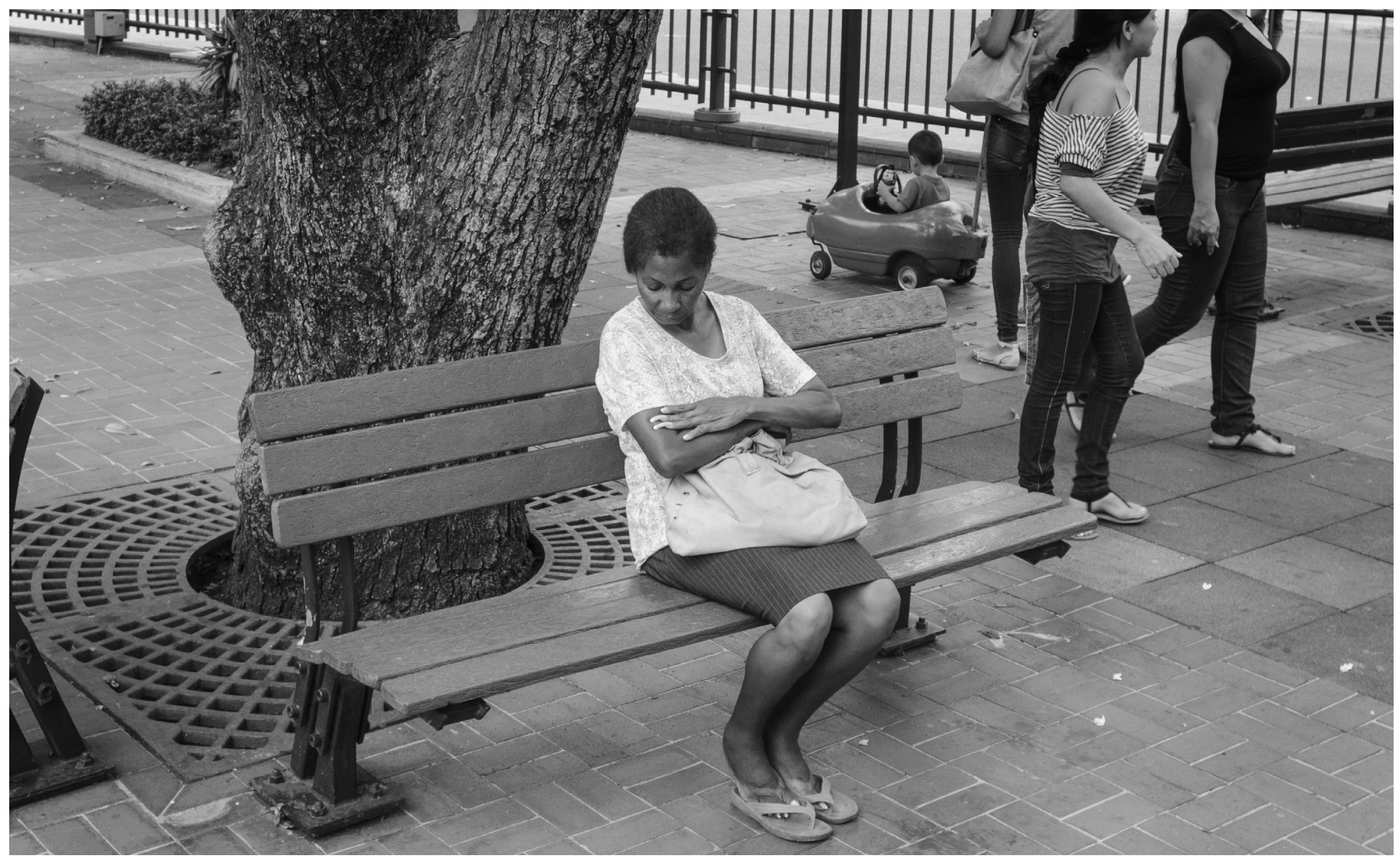

Foto: Cecilia Vidal

Esta dimensión está asociada con los aspectos estructurales que dificultan el acceso al tiempo libre y a diferentes maneras de socialización que acontecen en el espacio de la vida familiar y comunitaria. Este asunto aparece, por ejemplo, en el relato de una de las entrevistadas:

Para poder tener un buen empleo debes estar capacitado y desde ahí parte la desigualdad social. Fenotípicamente, los negros no podemos optar por trabajos a los que sí pueden los blancos. Podemos acceder a oficios varios y generales [relacionados con la servidumbre y el aseo]. Hago énfasis a nivel profesional, cuando se quieren destacar [los negros] y por su fenotipo no se los permiten, esto nos deja en marginalidad; no te miran las competencias, solo te miran y te descartan por el color de tu piel (Participante de Amafrocol, comunicación personal, 5 de agosto de 2018).

Aquí se puede ver cómo la injusticia de corte cultural, relativa a la clasificación del cuerpo según las expectativas de los grupos hegemónicos, se complementa con la lectura económica en la que dicha limitación se ubica en el acceso a oportunidades laborales.
La dimensión económica de la injusticia es la más detallada en las entrevistas realizadas en este estudio, debido a que se asocia la limitada oferta laboral con las experiencias de discriminación racial y de género, que son configuradoras de una norma social. Se trata de "una situación en la que personas con características similares en un sector $u$ ocupación son tratadas de manera desigual debido a sus rasgos observables como el sexo o la condición étnico-racial" (Gonzáles-Rivas, 2012, p. 564). Esto tiene como resultado una lectura del mundo laboral fundamentada en la desconfianza y en la prevención hacia la posibilidad de integración y participación en ambientes interculturales en los cuales pueda llevarse un desarrollo de los oficios o profesiones (Honneth, 2006).

Las injusticias económicas constituyen uno de los aspectos más visibles de las luchas sociales (González, 1997). La necesidad de redefinir los criterios empleados para la distribución de las riquezas y el modo en que esto influye en la convivencia se convierten en uno de los aspectos más considerados en el diagnóstico de las injusticias. 


\section{La dimensión política}

Los problemas relacionados con las limitaciones al reconocimiento cultural y a la redistribución equitativa de recursos económicos vienen asociadas con formas de invisibilización claramente identificables en el ámbito de la representación política. A juicio de Ibáñez (2010), “quien es pobre y mal valorado socialmente difícilmente puede realizarse plenamente en sus derechos políticos y decidir conjuntamente el destino de su sociedad" (p. 305).

La experiencia en las injusticias previas da lugar a la limitación en el espectro de la representación política, asunto que se vislumbra en el siguiente relato:

A nivel macro, el no reconocimiento nos afecta a nivel social, político, etc. Al Estado no le conviene que seamos reconocidos. Al ser una minoría, tenemos menos asignaciones en los planes de desarrollo, menos participación en la política.... Eso es invisibilización, no reconocimiento, y todo eso aporta a que ni siquiera tengamos agua potable o energía. Porque somos minoría, no necesitamos tanta plata (Participante de Amafrocol, comunicación personal, 10 de agosto de 2018).

Las injusticias de carácter político están asociadas con las limitadas posibilidades de representación en el espacio de los escenarios de disertación o reflexión de la vida en común (Fraser, 2008). La invisibilización resultante de todas las dimensiones de la injusticia produce una negación incuestionada de la posibilidad que tiene la gente para hacer parte de procesos de participación social que dan contenido a la vida democrática.

Siguiendo a Pécaut (2015), el auge de los grupos de narcotraficantes en el departamento del Valle del Cauca, cuya capital es la ciudad de Cali, trajo consigo un deterioro de la posibilidad que tenían las agremiaciones sociales, particularmente las campesinas, para participar en el entramado político de la región. En el caso de la historia de la violencia colombiana, uno de los aspectos más sobresalientes que ilustra esta dimensión está asociado con el modo en que los ordenamientos sociales del conflicto armado han transformado la sociabilidad que compone la vida ciudadana (Bejarano, Echandía, Escobedo, y Queruz, 1997).

En definitiva, el diagnóstico de las injusticias propuesto a partir de la reflexión de Fraser tiene como finalidad intervenir en el ámbito institucional de las interaccione sociales. De ahí que las experiencias objetivas de injusticia, que se desprenden de una manera de concebir la historia de la violencia colombiana, constituyan un campo de conocimiento dirigido a la concreción de gramáticas morales que giran en torno a la inclusión social y no se quedan exclusivamente en el plano psicológico del no reconocimiento. En tal sentido, es importante rastrear las representaciones fallidas, aquellas donde, a pesar de los intentos que posibilitan la participación, no es posible consolidar representación porque las reglas procedimentales niegan a unos la participación plena para producirse una representación fallida ordinaria (Ibáñez, 2010). Desde esta perspectiva, la inclusión en el espectro institucional se convierte en la finalidad sustancial de este tipo de diagnósticos.

Las mujeres participantes consideran dos escenarios fundamentales para subvertir las injusticias que padecen: por un lado, la cualificación para el trabajo y la educación universitaria; por otro, la visibilidad cultural mediante la celebración de las estéticas femeninas afrodescendientes a través de Makeda. De esta manera, educación y estética se convertirán en vehículos para subvertir las experiencias que han legitimado prácticas injustas que impiden la integración activa de las participantes en el escenario resolutivo de la vida intercultural en la ciudad. 


\section{El enmarcamiento social y las prácticas} de agenciamiento

El diagnóstico de las injusticias alcanza su madurez cuando los grupos pueden generar explicaciones propias de los fenómenos sociales que les ocasiona o genera vulnerabilidad social en el ámbito de la institucionalidad estatal. Se destaca el lugar que ocupan los procesos de comunicación en el intento por hacer comprensible el modo en que los grupos llevan a cabo la explicación sobre sus injusticias y las vías de solución que dan lugar a la experiencia del empoderamiento político (Jasper, 2012).

Para estudiar estos procesos de comunicación resulta sugerente incluir la noción de enmarcamiento social, entendida como un proceso prepolítico de objetivación de la experiencia social que da lugar a una organización social. Según Rivas (1998), los procesos de enmarcamiento ponderan las vivencias culturales estructuradoras de ideología con la lectura de las oportunidades del contexto social en el que se establece una crítica social. Se trata de formas de discursividad orientadas a producir síntesis relativas a los orígenes y el destino común de quienes comparten un mismo territorio u organización.

En este orden de ideas, es preciso advertir que el enmarcamiento es previo a la construcción de consensos políticos. Obedece a la constitución precedente del entramado de mensajes que se requieren para producir consignas verosímiles capaces de ordenar la acción social. Se trata también de una gramática moral para fundamentar una postura sobre los conflictos sociales (Honneth, 2009).

Se destaca el papel que tiene la sensibilidad moral en el tipo de enunciados que se producen para estructurar el enmarcamiento social. La moralidad, tal como es entendida en este estudio, refiere al raciocinio grupal sobre el trato o interacción que se estiman justos. Dicho de otra manera, la sensibilidad moral a la que se alude tiene que ver con el modo en que los grupos alcanzan ciertos grados de conciencia sobre las in- justicias comunes y, en consecuencia, pueden expresar valoraciones circunscritas en criterios de justicia (Habermas, 1999). Los criterios comunes que se desprenden de la legitimación de los procesos de comunicación orientan la composición del orden social.

El enmarcamiento es un ejercicio de definición de agendas en función de las exceptivas comunitarias de éxito. Siguiendo a Rivas (1998), es preciso rescatar el carácter dinámico en la fundamentación de una estrategia de comunicación exitosa. Lo que se busca en estos procesos es consolidar un conjunto de significados que puede hacer reconocible a un grupo de cara a otras colectividades que también luchan por el reconocimiento ante el Estado democrático de derecho. Este tipo de actividades se ubican en un plano metacomunicativo: la experiencia del enmarcamiento no se queda solo en la generación de información capaz de afectar el comportamiento a corto plazo, sino que se orienta a producir opinión pública, un asunto coherente con períodos largos de tiempo y capaces de producir cambios comportamentales (Rivas, 1998).

Los procesos de enmarcamiento social, en tanto que son configuradores de opinión pública, repercuten en la memoria social. Desde este enfoque hay que considerar la memoria como un proceso de generación de sentido que sirve para orientar la convivencia y producir generalidades sobre la vida social (Ricoeur, 1985/2004); es decir, principios de acción o eslóganes que, al ser comunes, legitiman y sintetizan el diagnóstico de las injusticias en virtud de su proyección social.

El enmarcamiento social está por fuera de los ámbitos hegemónicos en los que se construye el decir verdadero. No es su finalidad producir verdad histórica comprobable y verificable. De lo que se trata es de arraigar dispositivos capaces de dar sentido a las reivindicaciones y las estrategias organizativas que persiguen el éxito del cambio social. Por ello, de lo que trata es de producir verosimilitud: una ficción provisional que le compita 
a la verdad histórica, pero que pueda ser transformada constantemente según la lectura estratégica de las oportunidades y el modo en que se entienden los conflictos interculturales (Derrida, 2008).

Considerando el análisis propuesto, el enmarcamiento social se orienta a producir marcos de interpretación. Siguiendo a Goffman (citado por Rivas, 1998), tal enmarcamiento compone una agenda viva que constituye realidad social. Los marcos interpretativos que plantean los grupos, a partir del diagnóstico de las injusticias, tienen la capacidad de producir acción política en el entendido de que esta configura lenguajes expresivos variados y diversos. Sobre el asunto se presenta el siguiente relato:

A partir de saber cómo expresarme y comunicarme. Dar a entender a la gente con proyectos y demostraciones culturales. Vivir bien con los demás. Expresar la idea de que mi cultura tiene cosas muy positivas, lo negativo no fue porque quisimos, sino que nos obligaron. Una forma de hacerle entender a la gente sobre nuestra cultura es con Tejiendo Esperanzas [iniciativa de proyección social de Amafrocol a través de Makeda], esto le da a entender a la gente que nuestro cabello no es malo-quieto, al contrario, representa muchas cosas (Participante de Amafrocol, comunicación personal, 10 de agosto de 2018).

Lo que se puede apreciar en el caso de las mujeres de Amafrocol es un ejercicio de reflexión política que usa como recurso atributos de la estética femenina de las mujeres afrodescendientes del Pacífico colombiano. Esto les permite llevar a cabo un proceso de enmarcamiento social en el cual reflexionan sobre la memoria para componer los efectos que produce la injusticia en la vida de las participantes. De esta forma, la recuperación de los peinados típicos y las narrativas vinculadas a ello permiten cohesionar y orientar la discusión sobre el rol de las mujeres negras en la vida social vallecaucana.
El empoderamiento de ser mujer negra se logra con educación y sensibilización, esto es importante para que nos puedan representar en la política. Creemos que no basta con la mera representación; el solo hecho de que empieces a usar tu cabello tal como salió, dejar tu piel tal como es, usar el vestuario que hace referencia a tu cultura, todo eso es suficiente para representar una comunidad (Participante de Amafrocol, comunicación personal, 10 de septiembre de 2018).

Como se mencionó, la educación y la estética son los atributos de la experiencia del enmarcamiento social para el caso estudiado. La educación en asuntos como la historia de los movimientos afrocolombianos y las perspectivas feministas dotan a las participantes de contenidos que les permiten la experimentación y creación de lenguajes para configurar mensajes. Por su parte, la reivindicación de las estéticas femeninas permite presentar una manera de concebir la interacción social a partir de los referentes de origen en los que se relaciona el grupo con una manera de agenciar su participación social. Estos aspectos son coherentes con el pensamiento estratégico que se requiere para participar en los conflictos sociales.

La perspectiva de estudios implementada se vincula con la trayectoria de investigaciones sobre los movimientos sociales, en particular porque el interés está puesto en el estudio de la dimensión cultural de la acción colectiva (Touraine, 2013). Por consiguiente, son los ordenamientos simbólicos los que se transforman cuando las luchas por el reconocimiento cambian el sentido que se otorga a las interacciones sociales. Por ello, la interpretación que se realice de las experiencias de injusticia tiende a generar condiciones de partida para producir una deliberación pública de los asuntos comunes (Krotz, 2002). Al respecto puede destacarse el siguiente relato: 


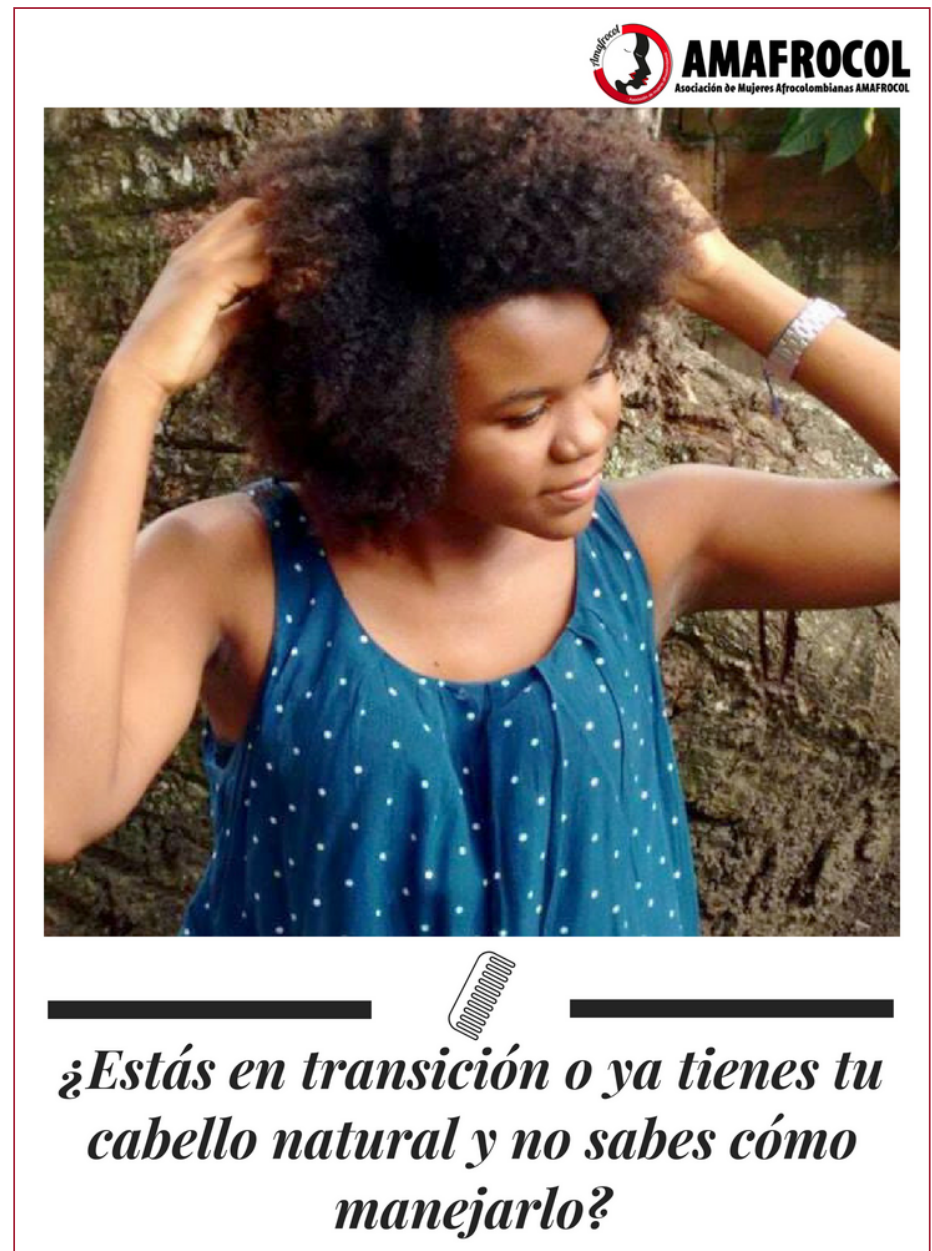

La hermosa Lina Lucumí de Bámbara, te asesorará mañana en la Gran Peinatón de Tejiendo Esperanzas. ¡No faltes!

Foto: Afiche realizado y diagramado por Sofía Arias Alonso

Casi siempre, en la comunidad más vulnerable estamos los afros, somos la comunidad que menos oportunidades tiene, la que está en las partes con menos recursos, donde no hay ingresos por parte del Estado. Entonces, van a haber menos oportunidades de educación y empleo, si no tenemos educación, pues menos oportunidades tenemos de salir adelante (Participante de Amafrocol, comunicación personal, 13 de julio de 2018).

El enmarcamiento social de los grupos históricamente subordinados constituye una evidencia de agenciamiento popular. Con esto se hace referencia a los liderazgos orgánicos que van resultando de la parti- cipación en el diagnóstico de las injusticias y la estructuración de los procesos de organización social. Según Cuellar y Zea (2014), el agenciamiento supone una manera de interacción y comportamiento social dirigido a la transformación moral de los individuos y la ponderación de sus fines comunes. El agenciamiento propuesto se resume en un compromiso cívico que intenta recomponer y circunscribir a los grupos a una esfera de reconocimiento institucional diversa y plural. Como se expresa en el siguiente relato:

Una de las formas que rescatamos es la identidad: de dónde venimos y lo que hacemos. Los proyectos culturales y sociales son los que nos ayudan a rescatar nuestras costumbres. Se da un mensaje a las personas sobre por qué hay este tipo de comportamientos. Todo esto ayuda a replicar la identidad negra (Participante de Amafrocol, comunicación personal, 1 de julio de 2018).

La noción de agenciamiento, entonces, rescata la disposición al consenso. Los actores sociales que participan en el entramado del enmarcamiento social han privilegiado un diagnóstico de las injusticias que genera cohesión entre sus miembros, produce una experiencia de organización capaz de generar opinión pública y tiene la potestad de producir consensos que, siendo limitados y temporales, otorgan proyección social a la agremiación.

Cabe destacar el carácter frágil de estos consensos, en tanto permiten pensar que la realidad social no es estática y siempre está vinculada a procesos reiterados de construcción de sentido (Botero, 2017). De ahí que el estudio de los procesos de comunicación bajo la forma de los enmarcamientos sociales permita entrever cómo se consolida la organización social en los tiempos en los que Colombia intenta abrirse a la construcción de paz, escenario relevante para los grupos étnicamente diferenciados. 


\section{Conclusiones}

A lo largo de este trabajo se ha propuesto un análisis sobre el papel que tiene el diagnóstico de las injusticias en la producción de ordenamiento social. Se ha constatado que las experiencias participativas originadas en este diagnóstico son generadoras de identidad y dan lugar a la aparición de experiencias prepolíticas, que fueron abordadas aquí desde la denominación de enmarcamiento social. Empezar por las injusticias implica pensar la posibilidad de instrumentalización de las identidades (Spivak, 2003); es decir, considerar que la lectura de las injusticias puede ser aprovechable como discurso común para ponderar fines socialmente relevantes en el ámbito de la lucha social por el reconocimiento, en la que participan otros grupos y las entidades del Estado (Pécaut, 2017).

En el caso de Amafrocol se puede apreciar que la superación de las injusticias produce motivaciones que tienen efectos en la capacidad de agenciamiento con la cual se desarrolla la proyección social de sus identidades políticas. De este modo, la principal injusticia cultural la constituye el racismo y las luchas de clases que componen ese fenómeno social. El esquema de clasificaciones producido por el racismo causa una afectación en las oportunidades laborales y el acercamiento a trabajos y oficios que han sido asociados con grupos mestizos. Se destaca asimismo el lugar que tienen los peinados y la estética femenina asociada con un modo de subvertir las clasificaciones racistas y misóginas que afectan el desenvolvimiento social, laboral y político. Los peinados ofrecen una vía para posicionar una opinión sobre el racismo y sus externalidades, se vuelven un símbolo de resistencia al tiempo que una postura política.

Las entrevistadas aluden a la invisibilización de sus identidades y, especialmente, a cómo muchas mujeres afrodescendientes optan por maltratar sus cuerpos para vincularse al campo hegemónico organizado por el racismo. En ese entrecruce, entre las injusticias culturales y económicas, se propone una justificación a la organización social que admite la cimentación de diversas estrategias para producir representación política. Entre tales alternativas se encuentra la cualificación laboral de las mujeres y su inclusión a la educación superior. Se estima que a través de los procesos de enseñanza-aprendizaje de la historia del movimiento social afrocolombiano y el feminismo es posible dotar a las participantes de una perspectiva crítica que les permita optimizar una conciencia sobre las injusticias.

Para este grupo el enmarcamiento social acontece desde la necesidad de representación en la esfera pública, asunto que se logra a través de la reivindicación de las estéticas femeninas afro propuesta por Makeda. La participación en escenarios resolutivos de la vida en común, en los que se puedan vislumbrar diferentes maneras de considerar la lucha social, conlleva a mejorar las condiciones de representación que afectan la participación social de las mujeres negras en Cali.

Para las entrevistadas, la experiencia de la vulnerabilidad viene acompañada de las limitaciones que padecen las mujeres, negras y pobres, en los contextos que históricamente han impuesto una cultura machista, racista y misógina. Desde esta perspectiva, se promueven perspectivas de cambio social que tienden a desarraigar las desigualdades armoniosas con las que conviven las participantes y que naturalizan el trato injusto que reciben (Ortega, 2011). De ahí que la formación política relativa al diagnóstico de las injusticias y la cualificación laboral respondan a la necesidad de proveer a las mujeres de Amafrocol de un lugar para la deliberación pública de los asuntos comunes.

A través de Makeda, esta organización implementa talleres relacionados con la formación política. Se trata de experiencias de alfabetización, con fuerte vertiente feminista, en la que se concibe que los peinados típicos constituyen una manera de contar la importancia de 
las mujeres negras en la sociedad. Desde esa perspectiva estética es posible narrar cómo las luchas sociales contribuyen al ensanchamiento del lenguaje político que se emplea para narrar las convivencias y la clasificación de la diversidad cultural. En este marco se llevan a cabo experiencias de construcción de sentido que componen el enmarcamiento social.

En síntesis, durante la investigación se pudo constatar cómo el enmarcamiento social produce alternativas para abordar la convivencia desde la dimensión estética y política de los conflictos interculturales. Se destaca en particular de qué manera las participantes de Amafrocol han sido capaces de diagnosticar las injusticias que padecen en virtud de la cimentación de una identidad política dispuesta al diálogo y la composición de consensos. De esta manera, el éxito del enmarcamiento social se verá representado en qué tan eficaz ha sido el proceso de comunicación para producir cohesión e inteligibilidad en las consignas que explican el origen de las injusticias que padecen. La evaluación de la eficacia política de las acciones y estrategias será objeto de futuras investigaciones, que tendrían como punto de partida el carácter prepolítico del enmarcamiento social para generar lenguajes y experiencias expresivas que tienen lugar en la vida pública.

\section{Referencias}

Bejarano, J. A., Echandia, C., Escobedo, R., y Queruz, L. (1997). Colombia: Inseguridad, violencia y desempeño económico en las áreas rurales. Bogotá, Colombia: Universidad Externado de Colombia-Fondo Financiero de Proyectos de Desarrollo.

Berrío Puerta, A. (2006). La perspectiva de los nuevos movimientos sociales en las obras de Sydney Tarrow, Alain Touraine y Alberto Melucci. Estudios Politicos, 29, 218-236.

Botero, S. (2017). El plebiscito y los desafíos políticos de consolidar la paz negociada en Colombia. Revista de ciencia política (Santiago), 37(2), 369-388.

Bourdieu, P. (2010). Las estructuras sociales de la economía. Buenos Aires, Argentina: Ediciones Manantial.
Chaparro Escudeo, M. (2015). Del pensamiento de Luis Ramiro Beltrán a las Epistemologías de la liberación y la alteridad. Revista Internacional de Comunicación y Desarrollo, 3, 43-153.

Chihu, A., y López, A. (2004). El análisis de los marcos en la obra de William Gamson. Estudios sociológicos, 2(22), 435-460. Recuperado de https://www.redalyc.org/articulo.oa?id=59806507

Clastres, P. (1977). Society against the State. Oxford, Inglaterra: Basil Blackwell.

Comins Mingol, I. (2002). Reseña. Construyendo la Paz, una perspectiva interdisciplinaria y transdiciplinaria. Convergencia. Revista en Ciencias Sociales, 9(29), 321-336.

Constitución política de Colombia. (1991). Asamblea Nacional Constituyente, Bogotá, Colombia, 6 de julio de 1991. Recuperado de https://dapre.presidencia.gov.co/normativa/constitucion-politica

Cuellar, M., y Zea, L. (2014). Procesos de agenciamiento y configuración de subjetividades en el ejercicio del restablecimiento de los derechos de una comunidad victima del desplazamiento forzado (Tesis de maestría). Universidad Pedagógica Nacional, Bogotá, Colombia.

Derrida, J. (2008). El siglo y el perdón. Entrevista con Michael Wieviorka. En E. Madina, R. Mate, J. Mayorga, M. Rubio, J. A. Zamora, El perdón, virtud política. En torno a Primo Levi (pp. 113-139). Barcelona, España: Anthropos.

Elias, N. (1998). La civilización de los padres y otros ensayos. Bogotá, Colombia: Norma.

Fraser, N. (1996). Redistribución y reconocimiento: Hacia una visión integrada de justicia de género. Revista Internacional de Filosofía Política, 8, 18-40.

Fraser, N. (1997). Iustitia interrupta. Reflexiones críticas desde la posición "postsocialista". Bogotá, Colombia: Siglo del Hombre.

Fraser, N. (2008). Escalas de justicia. Barcelona, España: Herder Editorial.

Gledhill, J. (2000). El poder y sus disfraces: perspectivas antropológicas de la política. Barcelona, España: Bellaterra.

Gonzáles-Rivas, N. (2012). Discriminación salarial: un análisis entre mujeres afrocolombianas y no afrocolombianas en el área metropolitana de Cali. Revista Latinoamericana de Ciencias Sociales, 1(10), 563-578. Recuperado de http://www.scielo. org.co/pdf/rlcs/v10n1/v10n1a36.pdf 
González, F. (1997). Aproximación a la configuración política colombiana. Controversia, 153(4), 19-72.

González, F. (2016). Poder y violencia en Colombia. Bogotá, CoIombia: Gente Nueva.

Grueso, D. I. (2009). Identidades étnicas, justicia y política transformativa. En G. Castellanos, D. I. Grueso, y M. Rodríguez, Identidad, cultura y política: perspectivas conceptuales, miradas empíricas (pp. 283-307). Cali, Colombia: Universidad del Valle.

Habermas, J. (1999). La inclusión del otro. Estudios de teoría política. Barcelona, España: Paidós.

Honneth, A. (1997). La lucha por el reconocimiento. Por una gramática de los conflictos morales. Barcelona, España: Grijalbo.

Honneth, A. (2006). Redistribución como reconocimiento: respuesta a Nancy Fraser. En N. Fraser, y A. Honneth, ¿Redistribución o reconocimiento? (pp. 89-148). Madrid, España: Morata.

Honneth, A. (2009). Patologías de la razón, historia y actualidad de la teoría crítica. Madrid, España: Katz.

Honneth, A. (2011). La sociedad del desprecio. Madrid, España:Trotta.

Ibáñez, F. (2010). Nancy Fraser: Escalas de justicia. Areté, Revisa de Filosofía, 2(22), 303-310. Recuperado de http://revistas. pucp.edu.pe/index.php/arete/article/view/126/125

Jasper, J. (2012). ¿De la estructura a la acción? La teoría de los movimientos sociales después de los grandes paradigmas. Sociológica, 27(75), 7-48.

Krotz, E. (2002). La otredad cultural entre utopía y ciencia - un estudio sobre el origen, el desarrollo y la reorientación de la antropología. México D. F., México: Fondo de Cultura Económica.

Moncayo, V. M. (2015). Hacia la verdad del conflicto: insurgencias guerrilleras y orden social vigente. Recuperado de http://www.altocomisionadoparalapaz.gov.co/ mesadeconversaciones/PDF/hacia-la-verdad-del-conflicto-insurgencia-guerrillera-y-orden-social-vigente-1447179178-1460343050.pdf

Ortega, F. A. (2011). El trauma social como campo de estudio. En F. A. Ortega, K. Erikson, J. A. Neil, J. Smelser, y R. Leys, Trauma, cultura e historia: Reflexiones interdisciplinarias para el nuevo milenio (pp. 17-63). Bogotá, Colombia: Universidad Nacional de Colombia.
Pécaut, D. (2015). La experiencia de la violencia: los desafíos del relato y la memoria. Medellín, Colombia: La Carreta Editores.

Pécaut, D. (2017). Daniel Pécaut. En busca de la nación colombiana. Conversaciones con Alberto Valencia. Bogotá, Colombia: Penguin Random.

Ricoeur, P. (2004). Tiempo y Narración I. México D. F., México: Siglo del Hombre. (Trabajo original publicado en 1985)

Rivas, A. (1998). El análisis de marcos: Una metodología para el estudio de los movimientos sociales. En P. Ibarra, y B. Tejerina, Los movimientos sociales (pp. 181-218). Madrid, España: Trotta.

Rodríguez, R. (2015). Pobreza, tragedia, gobierno. El caso del barrio El Retiro (Distrito de Aguablanca, Cali, Colombia) en los años 80. Revista O Social em Questão, 33(2), 195-214. Recuperado de http://osocialemquestao.ser.puc-rio.br/cgi/ cgilua. exe/sys/start.htm?infoid=386ctsid $=44$

Spivak, G. C. (2003). ¿Puede hablar el subalterno?. Revista Colombiana de Antropología, 39, 297-364.

Tobar Tovar, C. (2015). Algunas ideas sobre la relación entre consumo cultural, capital cultural y creación musical juvenil en la comuna 15 del Distrito Especial de Aguablanca en la ciudad de Cali, Colombia. Revista Periferia, 20(2), 195-214. https://doi.org/10.5565/rev/periferia.468

Torrico Villanueva, E. (2004). Comunicar la democracia: un aporte desde la academia. Revista Latinoamericana de Ciencias de la Comunicación, 1(1), 86-96.

Touraine, A. (2013). ¿Podremos vivir juntos?. Barcelona, España: Fondo de Cultura Económica.

Villafuerte, L. (2008). Una metodología interpretativa para el estudio de los movimientos sociales. Enmarcamientos y cultura. Una visión desde México. Revista Historia de la Educación Latinoamericana, 11, 225-246. Recuperado de http://www. redalyc.org/articulo.oa?id=86912618012

\section{Contribución autoral}

La concepción del trabajo científico fue realizada por Sofía Arias Alonso y Carlos Andrés Tobar Tovar. La recolección, interpretación y análisis de datos estuvo a cargo de Sofía Arias Alonso. La redacción y revisión del manuscrito fue realizada por Sofía Arias Alonso y Carlos Andrés Tobar Tovar. Ambos autores revisaron y aprobaron el contenido final. 